\title{
ACRL Midwinter meetings in Washington D.C.
}

\section{A tentative schedule}

G

d. note: AI A's Midwinter Meeting takes place from January $12-17,2001$, in Washington, D.C. This list of meeting times was current as we went to press. Be sure to check the program book for final times and locations of meetings.

\section{ACRL Board of Directors}

Board Orientation: Thursday, Jan. 11, 3:00-6:00 p.m.

Leadership Council: Friday, Jan. 12, 2:00-4:00 p.m.

First meeting: Sunday, Jan. 14, 2:00-4:00 p.m. Joint meeting with Budget \& Finance Committee: Monday, Jan. 15, 8:00-9:00 a.m.

Second meeting: Tuesday, Jan. 16, 1:004:30 p.m.

\section{ACRL General}

Leadership Council: Friday, Jan. 12, 2:00-4:00 p.m.

Presidential Candidate's Forum: Sunday, Jan. 14, 12:00-2:00 p.m.

President's Program Discussion Group: Monday, Jan. 15, 9:30-11:00 a.m. Topic: "Building Communities@ Your Library"

Scholarly Publishing and Academic Resources Coalition (SPARC)/ACRL Create Change Forum: Saturday, Jan. 13, 9:3011:00 a.m.

Scholarly Publishing and Academic Resources Coalition (SPARC)/ACRL Steering Committee: Sunday, Jan. 14, 2:00-4:00 p.m.

\section{ACRL Divisional Committees}

Academic/Research Librarian of the Year Award Selection: Monday, Jan. 15, 2:00-4:00 p.m. (closed)
Appointments: Saturday, Jan. 13, 2:004:00 p.m.; Sunday, Jan. 14, 2:00-4:00 p.m. (closed)

Best Practices Project Team: Monday, Jan. 15, 9:30 a.m.-3:30 p.m.

Budget and Finance: Saturday, Jan. 13, 8:30 a.m.-12:30 p.m.; Sunday, Jan. 14, 8:00-11:00 a.m.; Monday, Jan. 15, 8:0011:30 a.m.

Bylaws Committee: Saturday, Jan. 13, 9:30-11:00 p.m.; Monday, Jan. 15, 9:30-11:00 a.m.

Conference Program Planning-San Francisco, 2001: Saturday, Jan. 13, 8:309:30 a.m.

Conference Program Planning-Atlanta, 2002: Saturday, Jan. 13, 2:00-3:00 p.m.

Copyright: Sunday, Jan. 14, 8:30-11:00 a.m.

Council of Liaisons: Saturday, Jan. 13, 12:00-2:00 p.m.

Doctoral Dissertation: Saturday, Jan. 13, 9:30 a.m.-12:30 p.m.

Effective Practices: Saturday, Jan. 13, 2:00-4:00 p.m.; Monday, Jan. 15, 9:30 a.m.12:30 p.m.

Ethics: Sunday, Jan. 14, 4:30-6:30 p.m.

Government Relations: Saturday, Jan. 13, 2:00-4:00 p.m.; Monday, Jan. 15, 9:30 a.m.-12:30 p.m.

Information Literacy Advisory: Saturday, Jan. 13, 12:30-2:00 p.m.

Information Literacy Competency Standards, Task Force: Sunday, Jan. 14, 8:00-11:00 a.m.

Institute for Information Literacy: Friday, Jan. 12, 2:00-5:30 p.m.; Sunday, Jan. 14, 8:00-11:00 a.m. 
Institute for Information Literacy Advisory Group: Friday, Jan. 12, 2:00-5:30 p.m.; Sunday, Jan. 14, 8:00-11:00 a.m.

Institute for Information Literacy Best Practices: Monday, Jan. 15, 9:00 a.m.-3:30 p.m.

Institute for Information Literacy Immersion Program Faculty Planning: Thursday, Jan. 11, 8:00 a.m.-5:30 p.m.

Intellectual Freedom: Saturday, Jan, 13, 2:00-4:00 p.m.

International Relations: Sunday, Jan. 14, 8:30-11:00 a.m.

Lazerow Fellowship Committee: Sunday, Jan. 14, 4:30-6:00 p.m. (closed)

Media Resources: Sunday, Jan. 14, 9:3011:00 a.m.

Membership: Sunday, Jan. 14, 4:30-5:30 p.m.

National Conference Executive Committee-Denver, 2001: Saturday, Jan. 13, 11:00 a.m. $-1: 30$ p.m.

National Conference Speaker Orientation-Denver, 2001: Saturday, Jan. 13, 1:00-3:00 p.m.

National Conference Subcommittees-Denver, 2001: Monday, Jan. 15, 8:30 a.m.-12:30 p.m.

Nominations, 2002: Saturday, Jan. 13, 9:30-11:00 a.m.

President's Program Planning, 2001: Saturday, Jan. 13, 9:30-11:00 a.m.; Monday, Jan. 15, 5:30-6:30 p.m.

President's Program Planning, 2002: Saturday, Jan. 13, 9:00 a.m.-5:30 p.m.; Monday, Jan. 15, 9:30 a.m.-12:30 p.m.

Professional Development: Saturday, Jan. 13, 9:30-11:00 a.m.; Focus Group: Friday, Jan. 12, 11:00 a.m. $-12: 30$ p.m.

Publications: Saturday, Jan. 13, 8:00-9:00 a.m.; Monday, Jan. 15, 8:30-11:00 a.m.

Racial and Ethnic Diversity: Saturday, Jan. 13, 9:30 a.m.-12:30 p.m.

Research: Saturday, Jan. 13, 2:00-4:00 p.m.

Scholarly Communications Task Force: Sunday, Jan. 14, 9:00-11:00 a.m

Section Newsletter Editors: Sunday, Jan. 14, 2:00-4:00 p.m.

Standards and Accreditation: Sunday, Jan. 14, 9:30-11:00 a.m.

Statistics: Friday, Jan. 12, 4:30-5:30 p.m.

Status of Academic Librarians: Hearings on drafts of "Faculty Status and Collec- tive Bargaining" and "Guideline on Collective Bargaining": Saturday, Jan. 13, 8:3011:00 a.m.; Sunday, Jan. 14, 8:30-11:00 a.m.

TLTC Task Force: Saturday, Jan. 13, 2:004:00 p.m.

\section{ACRL Chapters Council}

ACRL Presidential Candidates Forum: Sunday, Jan. 14, 12:00-2:00 p.m.

Business and Orientation: Sunday, Jan. 14, 8:30-11:00 a.m.

\section{ACRL Editorial Boards}

CHOICE: Sunday, Jan. 14, 8:30-11:00 a.m.

College \& Research Libraries: Saturday, Jan. 13, 11:30 a.m.-12:30 p.m.

College \& Research Libraries News: Sunday, Jan. 14, 8:30-11:00 a.m.

New Publications Advisory Board: Sunday, Jan. 14, 12:30-1:30 p.m.

Publications in Librariansbip: Saturday, Jan. 13, 2:00-4:00 p.m.

RBM: Sunday, Jan. 14, 2:00-4:00 p.m.

\section{ACRL Sections}

Sections Council: Friday, Jan. 12, 4:305:30 p.m.

\section{African-American Studies Librarians Section}

Executive: Saturday, Jan. 13, 9:30-11:00 a.m.

All Standing Committees: Saturday, Jan. 13, 8:00-9:00 a.m.

Business/Membership: Saturday, Jan. 13, 2:00-4:00 p.m.

Cataloging Issues Discussion Group: Saturday, Jan. 13, I1:30 a.m.-12:30 p.m.

\section{Asian, African and Middle Eastern Section}

Executive: Saturday, Jan. 13, 9:30 a.m.12:30 p.m.

Nominating Committee: Friday, Jan. 12, 11:30 a.m.-12:30 p.m.

Program Planning - 2001: Friday, Jan. 12, 11:30 a.m.-12:30 p.m.

\section{Anthropology and Sociology Section}

Executive: Saturday, Jan. 13, 8:00-9:00 a.m.; Monday, Jan. 15, 9:30 a.m. $-12: 30$ p.m. 
Anthropology Librarians Discussion Group: Sunday, Jan. 14, 4:30-5:30 p.m.

Bibliography: Saturday, Jan. 13, 9:3011:00 a.m.; Sunday, Jan. 14, 9:30-11;00 a.m. Conference Program Planning-San Francisco, 2001: Sunday, Jan. 14, 8:00-9:00 a.m.

Conference Program Planning-Atlanta, 2002: Monday, Jan. 15, 8:00-9:00 a.m.

Liaison: Saturday, Jan. 13, 9:30-11:00 a.m.
Membership: Sunday, Jan. 14, 2:00-4:00 p.m.

Nominating: Saturday, Jan. 13, 9:30-11:00 a.m.

Publications: Saturday, Jan. 13, 9:30-11:00 a.m.

Review and Planning: Saturday, Jan. 13, 9:30-11:00 a.m.

Sociology Librarians Discussion Group: Saturday, Jan. 13, 11:30 a.m.-12:30 p.m.

\section{ACRL Discussion Groups}

Ed. note: Section discussion groups are listed with their sections.

Alliance for New Directions in Teaching and Learning: Monday, Jan. 15, 2:00-4:00 p.m.

Australian and Canadian Studies: Monday, Jan. 15, 8:00-9:00 a.m. Topic: $A$ Sojoum Througb Australian Libraries.

Consumer and Family Studies: Sunday, Jan. 14, 4:30-5:30 p.m. Topic: Fulltext Databases Covering the Consumer and Family Studies Discipline.

Criminal Justice/Criminology: Sunday, Jan. 14, 12:30-2:00 p.m. Topic: Library Services in Criminal Justice and Criminology; Guest from the National Criminal Justice Reference (U.S. Department of Justice).

Electronic Reserves: Sunday, Jan. 14, 8:30-11:00 a.m.

Electronic Text: Sunday, Jan. 14, 2:004:00 p.m. Topic: Best Practices for XMLXSL for TEI and EAD.

Fee-based Information Service Centers in Academic Libraries (FISCAL): Saturday, Jan. 13, 2:00-4:00 p.m.; Sunday, Jan. 14, 9:30-11:00 a.m.

Heads of Public/Readers Services: Sunday, Jan. 14, 4:30-5:30 p.m. Topic: Marketing of Library Services to the Virtual $\mathrm{Pa}$ trons.

Library and Information Science Collections: Saturday, Jan. 13, 2:00-4:00 p.m. Topic: Distance Education.

Library Development: Sunday, Jan. 14, 8:30-11:00 a.m. Topic: Library Access for Donors, Annual Fund, Telephone Outreacb and Issues Related to Academic Libraries.

Medium-Sized Academic Libraries: Sunday, Jan. 14, 9:30 a.m,-1:00 p.m. Topic: Assessment Tools.
MLA International Bibliography in Academic Libraries: Saturclay, Jan. 13, 9:30 a.m. $-12: 30$ p.m.

Personnel Administrators and Staff Development Officers of Large Research Libraries: Saturday, Jan. 13, 9:30-11:00 a.m.; Sunday, Jan. 14, 9:3011:00 a.m.

Philosophy/Religion Discussion Group: Sunday, Jan. 14, 2:00-4:00 p.m. Topic: Pbilosopbical Maps: Finding Pbilosopby in all the Wrong Places: the Experience of the USGS Library.

Popular Culture and Libraries: Sunday, Jan. 14, 4:30-5:30 p.m. Topic: Popular Cultire, Politics, and the Library as a Public Institution.

President's Program Discussion Group: Monday, Jan. 15, 9:30-11:00 a.m. Topic: Building Communities@ Your Library.

Research Discussion Group: Saturday, Jan. 13, 8:00-10:00 p.m. Topic: The Balancing Act: Researcb, Publisbing, Assigned Responsibilities as a Librarian, Teacbing, and Professional Service.

Scholarly Publishing and Academic Resources Coalition (SPARC)/ACRL Create Change Forum: Saturday, Jan. 13, 9:30-11:00 a.m.

Sports and Recreation Discussion Group: Sunday, Jan. 14, 8:00-9:00 a.m.

Team-Based Organizations: Saturday, Jan. 13, 9:30-11:00 a.m.

Undergraduate Libraries: Monday, Jan. 15, 8:30-11:00 a.m. Topic: Undergraduate Libraries: Survival of the Fittest? . . Fastest? ... Smallest? . . Busiest? ... 


\section{Arts Section}

Executive and Membership: Sunday, Jan. 14, 1:30 a.m.-2:00 p.m. at National Gallery.

All Committees: Saturday, Jan. 13, 9:30 a.m. $-12: 30$ p.m.

Dance Librarians Discussion Group: Sunday, Jan. 14, 4:30-5:30 p.m.

Film/Broadcast Studies Librarians Discussion Group: Sunday, Jan. 14, 2:00-4:00 p.m.

Performing Arts Discussion Group: Sunday, Jan. 14, 8:00-9:00 a.m.

\section{Community and Junior College Libraries Section}

Executive: Saturday, Jan. 13, 11:30 a.m.12:30 p.m.; Monday, Jan. 15, 8:30-11:00 a.m. All Committees: Saturday, Jan. 13, 8:3011;00 a.m.

Standards: Friday, Jan. 12, 2:00-4:00 p.m.

\section{College Libraries Section}

Executive: Saturday, Jan. 13, 9:30-11:00 a.m.; Monday, Jan. 15, 2:00-4:00 p.m.

CLIP Notes: Saturday, Jan. 13, 11:30 a.m.12:30 p.m.

College Librarians and Staff Discussion Group: Sunday, Jan. 14, 4:00-5:30 p.m.

College Library Directors' Discussion Group: Sunday, Jan. 14, 2:00-4:00 p.m.

Communications: Saturday, Jan. 13, 2:004:00 p.m.

Conference Program Planning-San Francisco, 2001: Sunday, Jan. 14, 8:30-11:00 a.m.

Conference Program Planning-Atlanta, 2002: Saturday, Jan. 13, 2:00-4:00 p.m.

Continuing Education: Monday, Jan. 15, 9:30-11:00 a.m.

Leadership: Monday, Jan. 15, 9:30-11:30 a.m.

Membership: Monday, Jan. 15, 8:00-9:00 a.m.

Research for College Librarianship: Sunday, Jan. 14, 8:00-11:00 a.m. Topic: "1-800 Publish: Helplines for Advancing Yourself and the Profession"

Standards: Saturday, Jan. 13, 11:30 a.m.12:30 p.m.

\section{Distance Learning Section}

Executive: Monday, Jan. 15, 2:00-4:00 p.m.

All Committees: Sunday, Jan. 14, 8:30-11:00 a.m.

Discussion Group: Monday, Jan. 15, 8:3011:00 a.m.

\section{Education and Behavioral Sciences Section}

Executive: Friday, Jan. 12, 7:00-9:00 p.m.

Advisory Council: Sunday, Jan. 14, 8:3011:00 a.m.

Conference Program Planning-San Francisco, 2001: Sunday, Jan. 14, 4:30-5:30 p.m.

Conference Program Planning-Atlanta, 2002: Sunday, Jan. 14, 4:30-5:30 p.m.

Consolidated Committee Mecting (Curriculum Materials, Distinguished Librarian Award, Instruction for Educators, Membership and Orientation, and Reference Sources and Services): Saturday, Jan. 13, 9:30 a.m.12:30 p.m.

Current Topics Discussion Group: Saturday, Jan. 13, 2:00-4:00 p.m.

Curriculum Materials Centers Directory Revision (Ad Hoc): Sunday, Jan. 14, 2:004:00 p.m.

Curriculum Materials Centers Standards/ Guidelines Committee (Ad Hoc): Saturday, Jan. 13, 8:00-11:00 a.m.

Digital Publication of the CMC Management Guide (Ad Hoc): Saturday, Jan. 13, 2:00-4:00 p.m.

Gerontology: Saturday, Jan. 13, 11:30 a.m. $-12: 30$ p.m.

Historical Textbook and Curriculum Collections Directory (Ad Hoc): Sunday, Jan. 14, 2:00-4:00 p.m.

Psychology/Psychiatry: Saturday, Jan. 13, 8:30 a.m.-12:30 p.m.

Publications \& Communications: Saturday, Jan. 13, 8:00 a.m.-12:30 p.m.

Social Work/Social Welfare: Friday, Jan. 12, 4:30-6:30 p.m.

\section{Instruction Section}

Section Dinner: Friday, Jan. 12, 6:00-9:00 p.m. Les Halles, \$37. Contact Madeline Copp at copp@nadn.navy.mil to reserve a space.

Executive: Satuday, Jan. 13, 8:00-9:00 a.m.; Tuesday, Jan. 16, 9:30 a.m.-12:30 p.m.

Advisory Council: Saturday, Jan. 13, 9:30-11:00 a.m.; Monday, Jan. 15, 2:004:00 p.m.

Awards: Saturday, Jan. 13, 2:00-4:00 p.m.; Sunday, Jan. 14, 9:30-11:00 a.m. (closed)

Communication: Saturday, Jan. 13, 2:004:00 p.m.; Sunday, Jan. 14, 9:30-11:00 a.m.

Conference Program Planning-San Francisco, 2001: Saturday, Jan. 13, 2:00-4:00 p.m.; Sunday, Jan. 14, 9:30-11:00 a.m. 
Conference Program Planning-Atlanta, 2002: Saturday, Jan. 13, 2:00-4:00 p.m.; Sunday, Jan. 14, 9:30-11:00 a.m.

Discussion Forum: Sunday, Jan. 14, 4:305:30 p.m.

Education: Saturday, Jan. 13, 2:00-4:00 p.m.; Sunday, Jan. 14, 9:30-11:00 a.m.

Emerging Technologies in Instruction: Saturday, Jan. 13, 2:00-4:00 p.m.; Sunday, Jan. 14, 9:30-11:00 a.m.

Immersion Alumni: Sunday, Jan. 14, 9:3011:00 a.m.

Instruction for Diverse Populations: Saturday, Jan. 13, 2:00-4:00 p.m.; Sunday, Jan. 14, 9:30-11:00 a.m.

Management for Instruction Services: Saturday, Jan. 13, 2:00-4:00 p.m.; Sunday, Jan. 14, 9:30-11:00 a.m.

Membership: Saturday, Jan. 13, 2:00-4:00 p.m.; Monday, Jan. 15, 9:30-11:00 a.m.

Model Statement Revision Task Force: Saturday, Jan. 13, 2:00-4:00 p.m.; Sunday , Jan. 14, 9:30-11:00 a.m.

New Intern Orientation: Saturday, Jan. 13, 8:00-9:00 a.m.

Nominating, 2002: Saturday, Jan. 13, 2:00 4:00 p.m.; Monday, Jan. 15, 9:30-11:00 a.m. (closed)

Planning: Saturday, Jan. 13, 2:00-4:00 p.m.; Monday, Jan. 15, 9:30-11:00 a.m.

Policy: Saturday, Jan. 13, 2:00-4:00 p.m.; Monday, Jan. 15, 9:30-11:00 a.m.

Preconference Planning-Denver, 2001: Saturday, Jan. 13, 2:00-4:00 p.m.; Monday, Jan. 15, 9:30-11:00 a.m.

Preconference Planning-San Francisco, 2001: Saturday, Jan. 13, 2:00-4:00 p.m.; Monday, Jan. 15, 9:30-11:00 a.m.

Preconference Planning-Atlanta, 2002: Saturday, Jan. 13, 2:00-4:00 p.m.; Monday, Jan. 15, 9:30-11:00 a.m.

Research and Scholarship: Saturday, Jan. 13, 2:00-4:00 p.m.; Monday, Jan. 15, 9:30-11:00 a.m.

Teaching Methods: Saturday, Jan. 13, 2:004:00 p.m.; Monday, Jan. 15, 9:30-11:00 a.m.

\section{Law and Political Science Section}

All Committees: Saturday, Jan. 13, 1:003:00 p.m.

General Meeting: Saturday, Jan. 13, 3:00-4:00 p.m.

Library Instruction Discussion Group: Sunday, Jan. 14, 2:00-4:00 p.m.
Marta Lange/CQ Award: Sunday, Jan. 14, 11:30 a.m.-1:30 p.m. (closed)

\section{Literatures in English Section}

Executive: Saturday, Jan. 13, 2:00-4:00 p.m.; Monday, Jan. 15, 2:00-4:00 p.m.

General Membership Meeting: Sunday, Jan. 14, 2:00-5:30 p.m.

All Committees: Monday, Jan. 15, 9:30 a.m. $-12: 30$ p.m.

Nineteenth Century Discussion Group: Sunday, Jan. 14, 4:30-5:30 p.m.

Nominating: Monday, Jan. 15, 9:3011:00 a.m. (closed)

Reference Discussion Group: Sunday, Jan. 14, 9:30-11:00 a.m.

\section{Rare Books and Manuscripts Section}

Executive: Monday, Jan. 15, 8:00-11:00 a.m.

Bibliographic Standards: Saturday, Jan. 13, 8:30 a.m.-12:30 p.m.; Sunday, Jan. 14, 8:00-11:00 a.m.

Budget and Development: Saturday, Jan. 13, 2:00-4:00 p.m.

Conference Development: Sunday, Jan. 14, 8:30-11:00 a.m.

Conference Program Planning-San Francisco, 2001: Sunday, Jan. 14, 9:3011:00 a.m.

\section{Meet Didier and Spalding-the candidates for ACRL President}

Hear Elaine Didier and Helen Spalding, the official candidates for ACRL vice-president/president-elect, speak at the ACRL Presidential Candidates Forum on Sunday, January 14, 2001, 12:30-2:00 p.m. during the Midwinter Meeting in Washington, D.C.

Didier is the dean of the university library at Oakland University. Spalding is associate director of libraries at the University of Missouri, Kansas City.

The forum, organized by the ACRL Chapters Council, will be followed by a light lunch, sponsored by EBSCO Subscription Services. 
Curators and Conservators Discussion Group: Sunday, Jan. 14, 8:30-11:00 a.m.

Exhibition Catalog Awards: Saturday, Jan. 13, 8:30 a.m.-12:30 p.m. (closed); Sunday, Jan. 14, 8:30-11:00 a.m

Information Exchange: Sunday, Jan. 14, 4:30-5:30 p.m.

MARC for Special Collections Discussion Group: Sunday, Jan. 14, 2:00-4:00 p.m.

Manuscripts and Other Formats Discussion Group: Sunday, Jan. 14, 2:00-4:00 p.m.

Membership and Professional Development: Saturday, Jan. 13, 2:00-4:00 p.m.

Nominating: Saturday, Jan. 13, 11:30 a.m. $-12: 30$ p.m.

Preconference Program Planning - San Francisco, 2001: Saturday, Jan. 13, 8:30-11:00 a.m.

Preconference Program Planning-Atlanta, 2002: Saturday, Jan. 13, 11:30 a.m12:30 p.m.

Public Services Discussion Group: Sunday, Jan. 14, 2:00-4:00 p.m.

Publications: Saturday, Jan. 13, 2:00-4:00 p.m. Security: Sunday, Jan. 14, 2:00-4:00 p.m.

Seminars: Saturday, Jan. 13, 2:00-4:00 p.m.

\section{Slavic and East European Section}

Executive: Sunday, Jan. 14, 4:30-5:30 p.m.

Automated Bibliographic Control: Saturday, Jan. 13, 9:30-11:00 a.m.

Conference Program Planning-San Francisco, 2001: Sunday, Jan. 14, 8:00-9:00 a.m.

Continuing Education: Saturday, Jan. 13, 11:30 a.m.-12:30 p.m.

Electronic Resources Task Force: Sunday, Jan. 14, 2:00-4:00 p.m.

Newsletter: Sunday, Jan. 14, 9:30-11:00 a.m.

Preservation: Saturday, Jan. 13, 2:00-4:00 p.m.

\section{Science and Technology Section}

Council: Friday, Jan. 12, 8:00-10:00 p.m.; Monday, Jan. 15, 8:30-11:30 a.m.

Comparison of Science and Technology Libraries: Saturday, Jan. 13, 8:30-11:00 a.m.

Conference Program Planning, San Francisco-2001: Saturday, Jan. 13, 9:30 a.m12:30 p.m.

Conference Program Planning-Atlanta, 2002: Saturday, Jan. 13, 8:30 a.m.-12:30 p.m.
Continuing Education: Sunday, Jan. 14, 9:30-11:00 a.m.

Discussion Group Chairs: Saturday, Jan. 13, 11:30 a.m.-12:30 p.m.

Forum for Science and Technology Library Research: Saturday, Jan. 13, 2:00-4:00 p.m.

General Discussion Group: Saturday, Jan. 13, 2:00-4:00 p.m.

Government Information: Sunday, Jan. 14, 8:30-11:00 a.m.

Heads of Science and Technology Libraries Discussion Group: Sunday, Jan. 14, 8:0010:00 p.m.

Membership and Recruitment: Sunday, Jan. 14, 8:00-9:00 a.m.

Oberly Award: Saturday, Jan. 13, 8:0011:00 a.m. (closed)

Organization and Planning: Saturday, Jan. 13, 2:00-4:00 p.m.

Publications: Saturday, Jan. 13, 9:30-11:00 a.m.

Science and Technology Databases and Publisher/Vendor Relations Discussion Groups: Sunday, Jan. 14, 9:30-11:00 a.m.

Subject and Bibliographic Access: Saturday, Jan. 13, 11:30 a.m.-12:30 p.m.

\section{University Libraries Section}

Executive: Saturday, Jan. 13, 8:30-11:00 a.m.; Monday, Jan. 15, 8:30-11:00 a.m.

Communications: Saturday, Jan. 13, 11:30 a.m.-12:30 p.m.

Conference Program Planning-San Francisco, 2001: Sunday, Jau. 14, 9:3011:00 a.m.

Conference Program Planning-Atlanta, 2002: Saturday, Jan. 13, 11:30 a.m.-12:30 p.m.; Monday, Jan. 15, 11:30 a.m.-12:30 p.m.

Current Topics Discussion Group: Saturday, Jan. 13, 2:00-4:00 p.m.

Librarians in Higher Education and Campus Administration Discussion Group: Saturday, Jan. 13, 11:30 a.m.-12:30 p.m.

Organization and Bylaws: Sunday, Jan. 14, 9:30-11:00 a.m.; Sunday Jan. 14, 2:004:00 p.m.

Policy and Planning: Sunday, Jan. 14, 9:30-11:00 a.m.

Public Service Directors of Large Research Libraries Discussion Group: Sunday, Jan. 14, 2:00-4:00 p.m.

(continued on page 1030) 


\section{The gateway to a world of information}

In a changing world, vision, experience and reliability are the qualities you value in a partner. Whether it be the management of your subscriptions or the delivery of an article to your desktop, Swets Blackwell provides quality services to libraries and information centers all over the world.

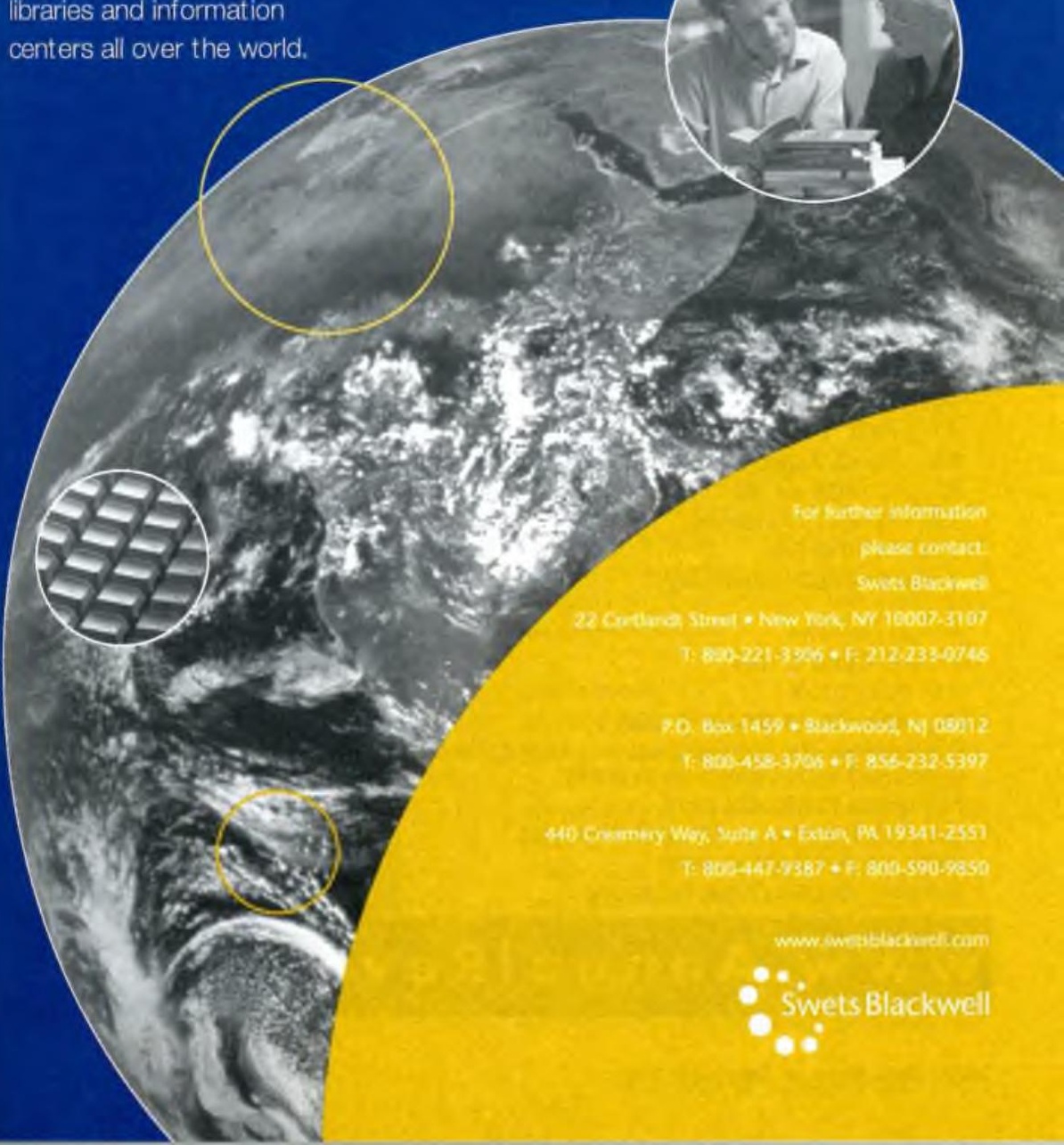

\section{Swets Blackwell}

Enhancing the power

of your knowledge 


\section{ANNUAL REVIEWS}

The Logical Starting Point

\section{Creating Site}

\section{License Solutions}

The Most Highly Cited Collection of Review Literature in the Biomedical, Physical, and Social Sciences.

\section{Visit Annual Reviews at the ACRL Show at Booth \#532!}

\section{Essential New Titles for Your Collection!}

Annual Review of Genomics and Human Genetics

Volume 1, August 2000

Annual Review of Biomedical Engineering

Volume 2, August 2000

Annual Review of Political Science

Volume 3, June 2000

For a Custom Site License Agreement or Consortium Pricing, Contact:

Andrea Lopez

Site License Account Manager

800.523 .8635 ext. 647 in USA/CAN

650.843.6647 Worldwide

650.424.0910 Fax

alopez@annualreviews.org

Annual Reviews

4139 El Camino Way

P.O. Box 10139

Palo Alto, CA 94303-0139 USA

Call Toll-free 800.523.8635 ext. 1 in USA/CAN or 650.493 .4400 Worldwide to order.

Fax orders to 650.424 .0910

Order Online at www.AnnualReviews.org email: service @ annurev.org

PRIORITY ORDER CODE: CRLN1200
29 Series to Link You to the World of Relevant Research

Anthropology

Astronomy \& Astrophysics Biochemistry

Biomedical Engineering Biophysics \& Biomolecular Siructure

Cell \& Developmental Biology

Earth \& Planetary Sciences

Ecology \& Systematics

Energy \& the Environment

Entomology

Fluid Mechanics

Genetics

Genomics \& Human Genetics

Immunology

Materials Science

Medicine

Microbiology

Neuroscience

Nuclear \& Particle Science

Nutrition

Pharmacology \& Toxicology

Physical Chemistry

Physiology

Phytopathology

Plant Physiology \& Plant Molecular Biology

Political Science

Psychology

Public Health

Sociology

\section{www. A n nulReviews.org}


("ACRL Midwinter Meetings . . " continued from page 1020)

Standards/Guidelines Review Committee: Saturday, Jan. 13, 11:30 a.m.-12:30 p.m.; Monday, Jan. 15, 2:00-4:00 p.m.

\section{Western European Studies Section}

Executive/General Membership: Tuesday, Jan. 16, 8:30 a.m.-12:30 p.m.

Cataloging Discussion Group: Sunday, Jan. 14, 4:30-5:30 p.m.

Classics, Medieval, and Renaissance Discussion Group: Saturday, Jan. 13, 2:00-4:00 p.m.

College and Medium-Sized Libraries Discussion Group: Saturday, Jan. 13, 11:30 a.m. $-12: 30$ p.m.

Conference Program Planning-San Francisco, 2001: Monday, Jan. 15, 9:3011:00 a.m.

Germanists Discussion Group: Sunday, Jan. 14, 9:30-11:00 a.m.

Membership: Saturday, Jan. 13, 9:3011:00 a.m.
Nijhoff Award: Saturday, Jan. 13, 9:3011:00 a.m. (closed)

Publications: Monday, Jan. 15, 2:00-4:00 p.m.

Research and Planning: Saturday, Jan. 13, 2:00-4:00 p.m.

Romance Languages Discussion Group: Sunday, Jan. 14, 4:30-5:30 p.m.

Scandinavian Discussion Group: Saturday, Jan. 13, 11:30 a.m.-12:30 p.m.

Social Sciences \& History Discussion Group: Monday, Jan. 15, 9:30-11:00 a.m.

\section{Women's Studies Section}

Executive: Monday, Jan. 15, 2:00-4:00 p.m.

All Committees: Sunday, Jan. 14, 9:3011:00 a.m.

Discussion Meeting: Monday, Jan. 15, 9:30-11:00 a.m.

General Membership: Sunday, Jan. 14, 4:30-5:30 p.m.

Research Committee (Ad Hoc): Monday, Jan. 15, 11:30 a.m. $-12: 30$ p.m.

\section{*ChoiceReviews.online It's more than just a database of reviews!}

$\star$ Access to some 70,000 reviews via the Internet

$\star$ E-mail notification of new reviews, based on individual subject interests

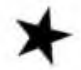

$\star$ Customized list creation, storage, and management

$\star$ Searchable database includes all CHOICE reviews since September 1988

$\star$ Password access allows high degree of customized use

$\star$ SUBSCRIBE NOW for $\$ 395$ ! Annual subscription includes personalized access for up to 20 users and all corresponding print issues of the magazine.

Sign up for a free trial today! www.ChoiceReviews.org

Phone: $(860) 347-6933 \times 23$

Fax: (860) 704-0465

E-mail: ChoiceOnline@ala-choice.org 100 Riverview Center, Middletown, CT 06457

\section{Booth 625}

American Library Association Midwinter Conference 